EXTENDED REPORT

\title{
Infliximab treatment for rheumatoid arthritis, with dose titration based on the Disease Activity Score: dose adjustments are common but not always sufficient to assure sustained benefit
}

\author{
P Sidiropoulos, G Bertsias, H D Kritikos, H Kouroumali, K Voudouris, D T Boumpas
}

Ann Rheum Dis 2004;63:144-148. doi: 10.1136/ard.2003.015933

See end of article for authors' affiliations

....................

Correspondence to:

Professor D T Boumpas,

Department of Internal

Medicine, Division of

Rheumatology, Clinical

Immunology and Allergy,

University of Crete,

Medical School, Voutes

71500, Heraklion,

Greece; boumpasd@

med.voc.gr

Accepted

23 September 2003
Background: Randomised controlled trials have shown that treatment with anti-tumour necrosis factor (anti-TNF) agents is effective in refractory rheumatoid arthritis (RA).

Objective: To determine the effectiveness of anti-TNF in a general unselected group of patients with refractory RA.

Methods: 68 patients with active RA despite treatment with disease modifying antirheumatic drugs were studied during 12 infliximab infusions. Infliximab ( $3 \mathrm{mg} / \mathrm{kg} /$ infusion) was given every 8 or 6 weeks. Clinical efficacy was assessed by the Disease Activity Score (DAS) index (44 joints). Dose adjustments were based on residual disease activity (DAS score $>2.4$ ). The primary end points were the percentage of patients achieving good or moderate response by the EULAR response criteria and the proportion of patients requiring dose adjustment.

Results: $20(29 \%)$ patients discontinued treatment owing to side effects, early inefficacy, or other considerations. Among the patients who continued treatment, $27(56 \%)$ and $32(67 \%)$ were responders on the 6th and 12th infliximab infusion, respectively. In the same patients, disease activity gradually improved without modifications in the initial dosing in $10(21 \%)$, whereas in $38(79 \%)$ the dose of infliximab and/or methotrexate was increased. Intensification of treatment led to a significant decrease in the mean DAS score in this group (from 5.27 just before dose modification to 4.54 before the 12 th infusion, $p<0.002$ ). The EULAR response category improved in only 10/38 (26\%), however.

Conclusions: In this initial observational study of patients with RA treated with recommended doses of infliximab, adjustments in treatment were common but not always sufficient to maintain adequate disease control. Longitudinal controlled trials are needed to define the optimal dose escalation in patients with suboptimal response.
$\mathrm{R}$ heumatoid arthritis (RA) is a chronic inflammatory arthropathy of substantial social and financial cost, and may reduce survival. ${ }^{1}$ Treatment of RA has changed following the clinical use of biologic therapies. Strategies that selectively target tumour necrosis factor $\alpha(\mathrm{TNF} \alpha)$ have shown efficacy when tested in patients with considerable disease activity despite treatment with disease modifying antirheumatic drugs (DMARDs). ${ }^{2-4}$

Infliximab is a chimeric monoclonal antibody that specifically binds both soluble and membrane bound TNF $\alpha$. It was the first anti-TNF $\alpha$ antibody that was clinically assessed for patients with RA. Several well designed randomised clinical trials (RCTs) have shown that infliximab has adequate clinical efficacy and an acceptable side effect profile..$^{5-8}$ Although the recommended dosing schedule for infliximab is at the lower end of doses that were evaluated in clinical studies, in the same studies higher doses and/or more frequent dosing suggested a potential for additional improvement. ${ }^{2}$ Based on these results and clinical experience, in May of 2002 a consensus statement on biological agents for treatment of RA stated that "in patients with an incomplete response, observations suggest that increasing the dose or reducing the dosing intervals may provide additional benefit, as may the addition or substitution of other DMARDs or other biological agents". . Although this is a sensible approach, its usefulness and the optimal escalation of treatment are not known.

Data from an RCT are of paramount importance to determine clinical efficacy of new treatments, but they have limitations related to their applicability in a general, unselected population. ${ }^{10}$ Observational studies and clinical protocols provide useful additional information about long term outcomes and side effects. Herein, we report our experience with the use of infliximab in patients with RA. More specifically, we report the long term efficacy, treatment failures, toxicity, and the application of validated tools for assessment of disease activity to modify treatment and the effect of treatment modification.

\section{METHODS}

Patients and treatment

Patients followed up by two rheumatology referral clinics (one in northern and the other in southern Greece) were included in this uncontrolled, open label study. They were recruited over a period of about 20 months and received infliximab because of considerable disease activity (Disease Activity Score (DAS) >3.7). Neither an upper limit for the steroid dose nor any limit for the current DMARDs was

Abbreviations: ACR, American College of Rheumatology; CRP, C reactive protein; DAS, Disease Activity Score; DMARD, disease modifying antirheumatic drug; ESR, erythrocyte sedimentation rate; $H A Q$, Health Assessment Questionnaire; PPD, purified protein derivative; RA, rheumatoid arthritis; $\mathrm{RCT}$, randomised controlled trial; $\mathrm{TB}$, tuberculosis; TNF $\alpha$, tumour necrosis factor $\alpha$; VAS, visual analogue scale 
applied. Patients received infliximab in the recommended dose of $3 \mathrm{mg} / \mathrm{kg}$ during weeks $0,2,6$, and thereafter every 8 or 6 weeks depending on individual response (see below) and were subsequently followed up for 12 infliximab infusions.

\section{Patient evaluation}

Patients were evaluated clinically at baseline and before each infusion. Routine laboratory tests such as complete blood count, erythrocyte sedimentation rate (ESR), C reactive protein (CRP), blood urea nitrogen, creatinine, liver function tests, and urine analysis were performed. A chest radiograph was performed on study enrolment. Patients recruited after November 2000 also had a skin test with purified protein derivative (PPD).

\section{Assessment of disease activity}

Disease activity was evaluated at study enrolment and before each of the 3rd to the 12th infliximab infusion using the following indexes: Ritchie articular index for tender joints, swollen joint count (44), visual analogue scale (VAS) for pain (0-10), VAS for patient's global assessment, doctor's global assessment on a Likert scale (1-5), and Health Assessment Questionnaire (HAQ). We applied the original DAS based on four variables (the Ritchie index, the 44 swollen joint count, the patient's assessment of disease activity, and the ESR) to assess disease activity on each visit. Response to treatment was assessed by the EULAR response criteria. According to these criteria patients were characterised as good, moderate, or non-responders based on both the DAS level attained and the change in DAS. ${ }^{11}$ Good response was defined as $>1.2$ improvement in the DAS from baseline and a DAS attained during follow up of $\leqslant 2.4$. Non-responders were patients with an improvement of $\leqslant 0.6$ or patients with an improvement of $>0.6$ but $\leqslant 1.2$ and a DAS attained during follow up of $>3.7$. The remaining patients were classified as moderate responders.

\section{Primary end points}

The primary end points included the proportion of patients who achieved good and moderate response after 12 infliximab infusions as well as the proportion of patients requiring dose adjustment.

\section{Adjustment in treatment}

Treatment modification (increase in the methotrexate dose or shortening the interval between infliximab infusions) to

Table 1 Baseline characteristics of patients $(n=68)$

\begin{tabular}{ll}
\hline Demographics & \\
Age (years) & $52.9(12)$ \\
Female, No (\%) & $51(75)$ \\
Disease duration (years) & $10.7(8.7)$ \\
Rheumatoid factor positive, No (\%) & $49(72)$ \\
Number of previous DMARDs & 2.6 \\
Concomitant treatment & \\
Concomitant methotrexate, No (\%) & $55(81)$ \\
Methotrexate dose (mg/week) & $13.0(3.5)$ \\
Concomitant corticosteroids, No (\%) & $41(60)$ \\
Corticosteroids dose (mg/day) & $9.1(4.5)$ \\
Disease characteristics & \\
Ritchie index & $28.1(14.3)$ \\
Swollen joints (44) & $19.7(11.3)$ \\
DAS & $5.4(1.5)$ \\
ESR (mm/1st h) & $44.3(25.6)$ \\
CRP (mg/l) & $29(36)$ \\
Haemoglobin (g/l) & $120(14)$ \\
Patient's global assessment (1-10) & $6.0(2.3)$ \\
Doctor's global assessment (1-5) & $3.1(0.8)$ \\
HAQ (0-3) & $1.4(0.6)$ \\
VAS for pain & $6.4(2.2)$ \\
\hline Values are presented as mean (SD) unless otherwise indicated. \\
\hline
\end{tabular}

Table 2 Withdrawals from the infliximab treatment

\begin{tabular}{lc}
\hline Reasons for withdrawal & Patients $(\%)$ \\
\hline Total & $20(29)$ \\
Lack of insurance coverage & $8(12)$ \\
Lack of efficacy & $5(7)$ \\
Infections & $4(6)$ \\
Severe allergic reactions & $2(3)$ \\
Haematological abnormalities (lymphocytosis) & $1(1)$ \\
\hline &
\end{tabular}

control disease activity was based predominantly on DAS at every visit (cut off point for low disease activity DAS $\leqslant 2.4$ ) and, in rare cases, on practical considerations, such as difficult access to the hospital or lack of insurance coverage. When adequate disease control was achieved corticosteroids were tapered. Treatment withdrawals were classified as withdrawals due to inefficacy, adverse reaction, or other cause.

\section{Statistical analysis}

Statistical evaluation within group was done by the use of paired Student's $t$ test. Rate differences between groups were analysed by the $\chi^{2}$ test, while comparison between groups was done by the Mann-Whitney test. A p value $<0.05$ (two tailed) was considered as significant.

\section{RESULTS}

Patients' demographics and disease characteristics A total of 68 patients received infliximab. Most had longstanding RA (mean disease duration 10.7 years; table 1), failed multiple DMARDs, and had functional status ranging from I to III according to the revised American College of Rheumatology (ACR) criteria. ${ }^{12}$ At study entry all but five patients (93\%) had a high level of disease activity (DAS >3.7). Consistent with that was the high number of swollen joints (mean (SD) $19.7(11.3)$ ) as well as the high Ritchie articular index score (mean (SD) 28.1 (14.3)).

Most patients received methotrexate before infliximab treatment either as monotherapy or in combination with different DMARDs. The majority of the patients $(55 / 68,81 \%)$ continued methotrexate upon study enrolment but discontinued other DMARDs. Six patients had contraindication to the use of methotrexate and received another DMARD (hydroxychloroquine (four), cyclosporin A (one), and hydroxychloroquine in combination with sulfasalazine (one)), while seven received monotherapy with infliximab. The majority of patients $(41 / 68,60 \%)$ on study enrolment received corticosteroids, with a mean (SD) dose of 9.1 (4.5) $\mathrm{mg}$ /day. Patients were followed up for up to 12

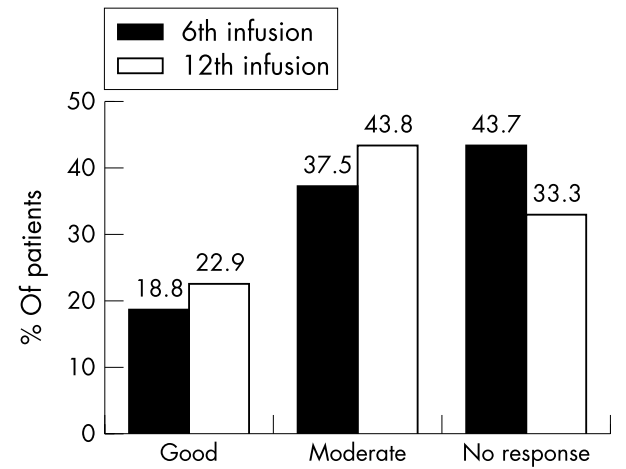

Figure 1 Response rates at the 6 th and 12 th infusions of infliximab according to EULAR response criteria (\% of patients). 


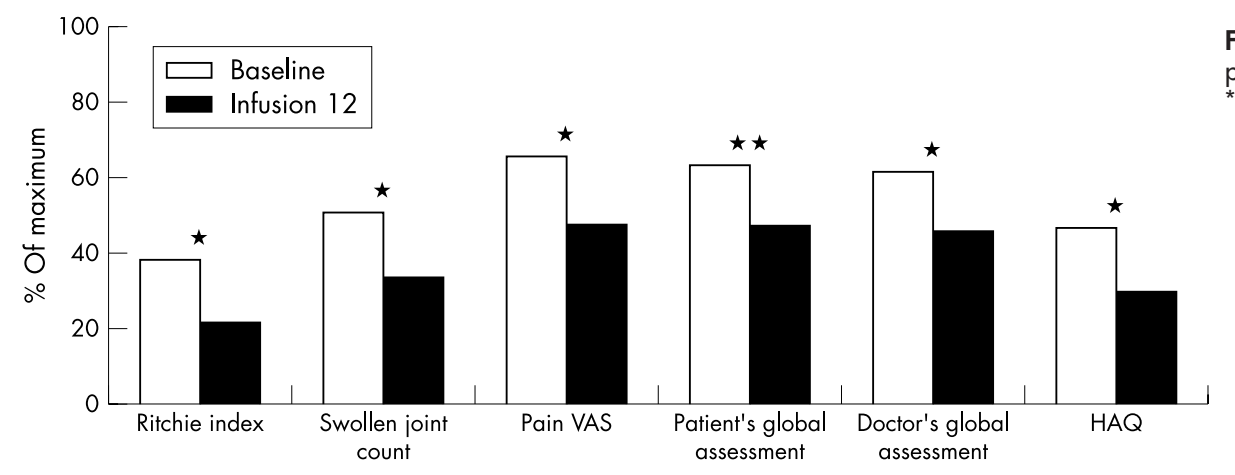

Figure 2 Improvement in clinical parameters $(n=48) .{ }^{*} \mathrm{p}<0.001$; ${ }^{* *} p<0.002$.

infliximab infusions. Because of modifications in the infliximab scheme the mean treatment duration was 72.6 weeks (range 54-78).

\section{Withdrawals}

Overall, $20(29 \%)$ patients discontinued treatment (table 2). All but two discontinuations (90\%) occurred during the first six infliximab infusions. The single late discontinuation was at the 10th infusion because of marked lympocytosis. All patients who discontinued treatment because of inefficacy had considerable disease activity with a DAS score of at least 3.7 and no improvement in any of the patient oriented or physician measures. All five patients who discontinued treatment because of inefficacy received no modification to the dose or scheme of treatment before discontinuation. This was because experience with infliximab treatment was limited during the first months of its clinical application. An additional four patients discontinued because of concurrent infection. Two of them had tuberculosis (TB), one lethal miliary $\mathrm{TB}$ and the other extrapulmonary $\mathrm{TB}$ with cervical lymph node involvement. Another patient after 60 weeks of treatment was diagnosed as having possible TB arteritis of the subclavian artery. The fourth patient had a severe pneumonia of possibly infectious aetiology. Two patients, both receiving monotherapy with infliximab, discontinued because of serious allergic reactions, one on the 5th and the other on the 6th infliximab infusion. All subsequent data analysis for efficacy and treatment modification are for the 48 patients who completed 12 infliximab infusions.

\section{Efficacy}

Efficacy was maintained through the treatment period with no statistically significant differences between the 6th and 12th infusion for the three categories of response (good, moderate, and no response). The percentage of responders (good plus moderate) at the 6th and 12th infusion was 56\% and $67 \%$ respectively (fig 1 ). All clinical parameters were significantly improved for those patients who completed the study (fig 2). However, only nine (19\%) patients could satisfy the remission cut off criterion (DAS <1.6) at least once during the treatment period and only three patients at the 12th infusion. Moreover, only $23 \%$ of the patients had DAS scores $<2.4$ (low disease activity cut off limit) at thel2th infusion.

The mean corticosteroid dose for those patients who completed 12 infusions decreased from $8.6 \mathrm{mg} /$ day at study entry to 7.1 and $6.0 \mathrm{mg} /$ day at the 6 th and 12 th infusion, respectively $(\mathrm{p}<0.05$ between study entry and both the 6th and 12th infusions), while $30 \%$ (9/30) were able to discontinue steroids (table 3 ).

\section{Dose adjustments}

Adjustment in the infliximab scheme or methotrexate was done in the majority of the patients as outlined in "Methods". Among the patients receiving methotrexate, the dose of methotrexate was increased in $20 \%$; the mean dose increased from 13.2 to $14.2 \mathrm{mg} / \mathrm{wk}$ between the 1st and 12th infusion, respectively (table 3 ). The frequency of infliximab infusions was increased to at least every 6 weeks in $73 \%$ of patients. The mean time point of infliximab dosing adjustment was the 8 th infusion (4th to 11 th; fig 3 ). In contrast, in $10(21 \%)$ patients adequate clinical response was achieved and in this group neither methotrexate nor infliximab doses were increased. When differences at baseline characteristics between the stable treatment group $(21 \%)$ and the dose adjustment group were assessed, it was shown that the mean DAS in the latter was significantly higher than in the first group (6.05 $v$ 4.57, $\mathrm{p}<0.01$; fig 3 ). No differences were detected in age, disease duration, sex, methotrexate, or corticosteroid dosages between these two groups.

\section{Response to dose adjustment}

In total, either methotrexate or infliximab dosing were modified in $38 / 48(79 \%)$ patients. The mean DAS for this group of patients significantly improved after treatment modification from 5.27 just before dose modification to 4.54 before the 12th infusion $(\mathrm{p}<0.002$; fig 3$)$. After either treatment modification the EULAR response category was improved in $10(26 \%)$ of these 38 patients, remained the same in $23(61 \%)$, and worsened in 5 (13\%) (fig 4$)$.

Table 3 Dose adjustment during treatment for those patients who completed 12 infliximab infusions

\begin{tabular}{lllll}
\hline Treatment & Baseline & Infusion No 6 & Infusion No 12 & p Value* \\
\hline Corticosteroids (mg/day, $\mathrm{n}=30$ ) & $8.6(3.5)$ & $7.1(2.9)$ & $6.0(2.1)$ & $<0.05$ \\
$\begin{array}{l}\text { Methotrexate (mg/week, } \mathrm{n}=42) \\
\text { Infliximab (\% of patients with shortened infusion }\end{array}$ & $13.2(4.3)$ & $13.6(3.9)$ & $14.2(3.5)$ & $<0.05$ \\
interval, $\mathrm{n}=48$ ) & 37.5 & 72.9 & \\
\hline
\end{tabular}

Results are shown as mean (SD).

${ }^{*} \mathrm{p}$ Value between baseline and the 12th infusion. 


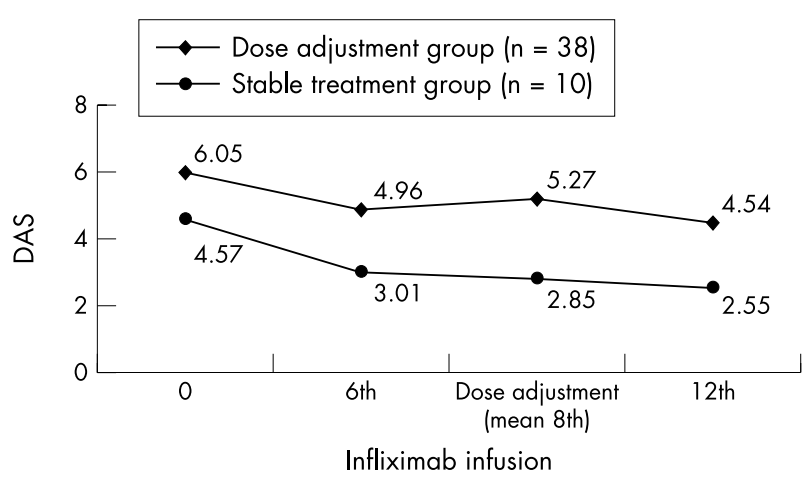

Figure 3 Mean DAS over time for each group of patients: the first with stable treatment $(n=10)$ and the second with treatment modification $(n=38)$.

\section{DISCUSSION}

RCTs are the "gold standard" for assessing clinical efficacy of a therapeutic intervention. However, application of these types of study to a chronic disease like RA has several limitations ${ }^{10}$ as a significant number of patients seen in routine care do not meet the criteria for inclusion, thus limiting the applicability of results in a limited number of patients. Long term observational studies and clinical protocols are of great value for obtaining data about effectiveness and long term prognosis of patients with RA. ${ }^{13}$ We consider that data presented as in this study are useful for a better and more reliable assessment of clinical results of recently developed therapeutic interventions for RA.

Although the size of the cohort was modest, we believe this is a group representative of a "real life" clinical practice. Thus, most of the patients had longstanding RA (mean disease duration 10.7 years) with multiple DMARD failure (mean 2.6) and considerable disease activity (mean DAS 5.4, with $93 \%$ of the patients having a DAS $>3.7$ ). Their high disease activity is indicated by the fact that the majority of them received corticosteroids at a mean dose of $9.1 \mathrm{mg} /$ day, which is more than the accepted dose for long term use in patients with RA. Two patients developed TB with an additional possible TB arteritis. A baseline PPD skin test was not available in these three patients. After recognition of the potential for reactivation of $\mathrm{TB}$, a PPD skin test was performed in all patients subsequently enrolled.

The ACR response criteria were originally developed to discriminate between active treatment and placebo and are used mainly in RCTs. ${ }^{14}$ We applied the DAS because we were also interested in actual disease activity status, an important consideration in clinical practice. Applying the EULAR response criteria we found that treatment effectiveness was sustained throughout the treatment period. The percentage of patients who were responders (good or moderate) was not significantly different between the 6th and 12th infusions (56\% and $67 \%$ at the 6 th and 12th infusion, respectively; fig 1). In agreement with previous experience, significant residual disease activity remained, even for responders (mean DAS of good and moderate responders at the 12th infusion $3.01)$. Moreover, only nine (19\%) patients entered remission $($ DAS $<1.6)$ at some time during treatment and three $(6 \%)$ of them just before the 12th infliximab infusion.

Because the ACR20 response criteria correspond roughly with the sum of good and moderate responders, response rates for our patients are comparable with response rates from clinical trials and with a registry reported recently from Sweden. ${ }^{15}$ Moreover, our treatment survival was around $70 \%$, which is also similar to that reported recently from the Swedish registry. However, the data on efficacy in this study

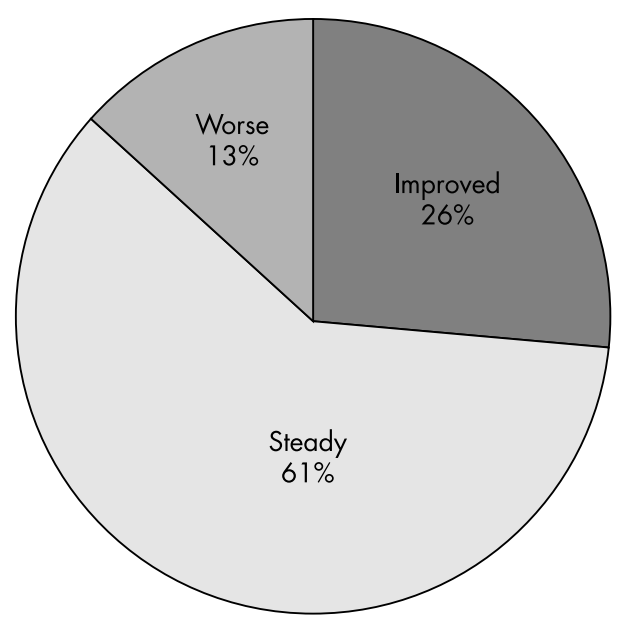

Figure 4 Changes in EULAR response categories after treatment adjustments $(n=38)$.

have to be interpreted with caution. Because this was an open label study, we cannot completely rule out the possibility that a placebo effect or regression to the mean might have influenced the response rates. However, the long duration of the study makes it unlikely that either of these had a substantial effect on the analysis (because the placebo effect usually declines with time), and regression to the mean would have a similar effect on both groups.

Two groups of patients can be distinguished based on treatment efficacy and subsequent need for an increase of either methotrexate or infliximab (fig 3). About $20 \%$ of the patients improved gradually and there was no need for modification in treatment schedule. This group of patients had significantly lower disease activity at the beginning than the second group (DAS $4.57 \vee 6.05, \mathrm{p}<0.05$ ), while no significant differences in either methotrexate or corticosteroid doses were detected between them. However, the majority of patients required dose modification at some point (4th to 11 th, mean 8th infliximab infusion) to control disease activity. Although DAS in this group improved significantly between baseline and the 12th infusion (from 6.05 to $4.54, \mathrm{p}<0.001$ ), they had more active disease than the first group at all times during the treatment period (6th, 8th, and 12 th infliximab infusion, $\mathrm{p}<0.01$ between groups).

Comparison of response rates just before treatment adjustment and before the 12th infliximab infusion showed that the majority of the patients remained at the same response category and only $26 \%$ improved response status (fig 4). It might be argued that the modifications of infliximab and/or methotrexate were not aggressive enough to improve optimally the disease activity level as assessed by the DAS index, or that disease in these patients is not strictly $\mathrm{TNF} \alpha$ dependent.

The immunogenicity of infliximab has been recently assessed in patients with Crohn's disease treated with infliximab. ${ }^{16}$ The authors concluded that concomitant immunosuppression prevents the formation of antibodies against infliximab, reducing the incidence of allergic reactions and increasing the duration of response. Thus, in patients with RA methotrexate acts both as a DMARD and by reducing infliximab immunogenicity. The finding in the present cohort that treatment modification is needed in the majority of the patients can be attributed to either a "low dose" infliximab scheme $(3 \mathrm{mg} / \mathrm{kg}$ ) or to an inadequate suppression of infliximab immunogenicity by methotrexate "low dosing" regimen (mean dose $13.2 \mathrm{mg} /$ week, lower than the $15 \mathrm{mg} /$ week in the aforementioned study). 
Den Broeder et al recently published their successful experience of dose titration with adalimumab, a human monoclonal anti-TNF $\alpha$ antibody, applying DAS28 to monitor disease activity. ${ }^{17}$ Our data support this approach of close monitoring of disease activity and subsequent dose adjustment, especially when recently developed and expensive agents like biological agents are used. To this end, efforts to develop a "physician friendly" Simple Disease Activity Index (SDAI) that retains the sensitivity assessment characteristics of the DAS and ACR response criteria, but is at the same time an easy way to assess disease activity in daily practice, are certainly useful. ${ }^{18}$

The goal of treatment of RA is to control inflammation, and regular disease activity monitoring is needed for clinical decision making. TNF $\alpha$ antagonists are potent and expensive drugs and their use in clinical practice requires close monitoring and application of validated tools such as the EULAR response criteria, which assesses both functional capacity and progression of joint damage, ${ }^{19}$ may facilitate this task. $^{20}$

\section{ACKNOWLEDGEMENTS}

This work was supported-in part-by an unrestricted grant from Schering Plough of Greece.

The authors thank Dr Marinela Mamoulaki, Panagiota Siaka, Giasna Kakavouli, Maria Kasapaki, RN, Sofia Sfakianaki, RN, and the nursing staff of the rheumatology clinics for patient care, and Dr Gabor Illei for critical review of the manuscript.

\section{Authors' affiliations}

P Sidiropoulos, G Bertsias, H D Kritikos, H Kouroumali, D T Boumpas, University Hospital, Medical School, University of Crete, Heraklion, Greece

P Sidiropoulos, K Voudouris, First General Hospital of Thessaloniki "Agios Pavlos", Thessaloniki, Greece

\section{REFERENCES}

1 Gabriel S, Crowson CS, Kremers HM, Doran MF, Turesson C, O'Fallon NM, et al. Survival in rheumatoid arthritis. a population based analysis over 40 years. Arthritis Rheum 2003:48:54-8.

2 Lipsky PE, van der Heijde DM, St Clair EW, Furst DE, Breedveld FC, Kalden JR, et al. Infliximab and methotrexate in the treatment of rheumatoid arthritis. N Engl J Med 2000;343: 1594-602.

3 Weinblatt ME, Kremer JM, Bankhurst AD, Bulpitt KJ, Fleischmann RM, Fox RI, et al. A trial of etanercept, a recombinant tumor necrosis factor receptor: $\mathrm{Fc}_{\mathrm{c}}$ fusion protein, in patients with rheumatoid arthritis receiving methotrexate [see comments]. N Engl J Med 1999;340:253-9.

4 Weinblatt ME, Keystone EC, Furst DE, Moreland LW, Weisman MH, Birbara CA, et al. Adalimumab, a fully human anti-tumor necrosis factor alpha monoclonal antibody, for the treatment of rheumatoid arthritis in patients taking concomitant methotrexate: the ARMADA trial. Arthritis Rheum 2003;48:35-45.

5 Elliott MJ, Maini RN, Feldmann M, Long-Fox A, Charles P, Katsikis P, et al. Treatment of rheumatoid arthritis with chimeric monoclonal antibodies to tumor necrosis factor alpha. Arthritis Rheum 1993;36:1681-90.

6 Elliott MJ, Maini RN, Feldmann M, Kalden JR, Antoni C, Smolen JS, et al. Randomised double-blind comparison of chimeric monoclonal antibody to tumour necrosis factor alpha (cA2) versus placebo in rheumatoid arthritis. Lancet 1994:344:1105-10.

7 Maini R, St Clair EW, Breedveld F, Furst D, Kalden J, Weisman M, et al. Infliximab (chimeric anti-tumour necrosis factor alpha monoclonal antibody) versus placebo in rheumatoid arthritis patients receiving concomitant methotrexate: a randomised phase III trial. ATTRACT Study Group. Lancet 1999;354:1932-9.

8 Maini RN, Breedveld FC, Kalden JR, Smolen JS, Davis D, Macfarlane JD, et al. Therapeutic efficacy of multiple intravenous infusions of anti-tumor necrosis factor alpha monoclonal antibody combined with low-dose weekly methotrexate in rheumatoid arthritis [see comments]. Arthritis Rheum 1998;41:1552-63.

9 Furst DE, Breedveld FC, Kalden JR, Smolen JS, Antoni CE, Biilsma JWJ, et al. Updated consensus statement on biological agents for the treatment of rheumatoid arthritis and other rheumatic diseases (May 2002). Ann Rheum Dis 2002;61(suppl II):ii2-7.

10 Pincus T. Limitations of randomized controlled clinical trials to depict accurately long-term outcomes in rheumatoid arthritis. Z Rheumatol 1998;57:46-9.

11 van Gestel AM, Prevoo ML, van 't Hof MA, van Rijswijk MH, van de Putte LB, van Riel PL. Development and validation of the European League Against Rheumatism response criteria for rheumatoid arthritis. Comparison with the preliminary American College of Rheumatology and the World Health Organization/International League Against Rheumatism Criteria. Arthritis Rheum 1996;39:34-40

12 Hochberg MC, Chang RW, Dwosh I, Lindsey S, Pincus T, Wolfe F. The American College of Rheumatology 1991 revised criteria for the classification of global functional status in rheumatoid arthritis. Arthritis Rheum 1992;35:498-502.

13 Hawley DJ, Wolfe F. Are the results of controlled clinical trials and observational studies of second line therapy in rheumatoid arthritis valid and generalizable as measures of rheumatoid arthritis outcome: analysis of 122 studies. J Rheumatol 1991;18:1008-14.

14 van Riel PL, van Gestel AM. Clinical outcome measures in rheumatoid arthritis. Ann Rheum Dis 2000;59(suppl I):i28-31.

15 Geborek P, Crnkic M, Petersson IF, Saxne T. Etanercept, infliximab, and leflunomide in established rheumatoid arthritis: clinical experience using a structured follow up programme in southern Sweden. Ann Rheum Dis 2002;61:793-8.

16 Baert F, Noman M, Vermeire S, Van Assche G, D' Haens G, Carbonez A, et al. Influence of immunogenicity on the long-term efficacy of infliximab in Crohn's disease. N Engl J Med 2003;348:601-8.

17 Den Broeder AA, Creemers MC, van Gestel AM, van Riel PL. Dose titration using the Disease Activity Score (DAS28) in rheumatoid arthritis patients treated with anti-TNF-alpha. Rheumatology (Oxford) 2002;41:638-42.

18 Smolen JS, Breedveld FC, Schiff MH, Kalden JR, Emery P, Eberl G, et al. A simplified disease activity index for rheumatoid arthritis for use in clinical practice. Rheumatology (Oxford) 2003;42:244-57.

19 Welsing PM, van Gestel AM, Swinkels HL, Kiemeney LA, van Riel PL. The relationship between disease activity, joint destruction, and functional capacity over the course of rheumatoid arthritis. Arthritis Rheum 2001;44:2009-17.

20 Fransen J, Stucki G, van Riel P. The merits of monitoring: should we follow all our rheumatoid arthritis patients in daily practice? Rheumatology (Oxford) 2002;41:601-4. 